\title{
The Role of Clinical Pharmacist in Pharmacovigilance
}

\author{
Asim Elnour ${ }^{1^{*}}$ and Farah Hamad ${ }^{2}$ \\ ${ }^{1}$ Pharmacology Department, United Arab Emirates University (UAEU), United Arab Emirates \\ ${ }^{2}$ Ajman University of Sciences and Technology, United Arab Emirates
}

"Corresponding author: Asim Elnour, Pharmacology Department, Faculty of Medicine and Health Sciences (FMHS), United Arab Emirates University (UAEU), United Arab Emirates, Tel: 050-6734096; E-mail: assahura1962@uaeu.ac.ae

Received date: September 01, 2014, Accepted date: September 03, 2014, Published date: September 10, 2014

Copyright: (C) 2014 Elnour A, et al. This is an open-access article distributed under the terms of the Creative Commons Attribution License, which permits unrestricted use, distribution, and reproduction in any medium, provided the original author and source are credited.

Keywords: Clinical pharmacist; Pharmacovigilance; Clinical; Health Information System

\section{Editorial}

The clinical pharmacist professional is enormously growing worldwide. The need for the practicing clinical pharmacist became an inevitable in all healthcare settings. This gain of reputation and recognition has come from the emerging needs for safe, economic and rational use of pharmaceuticals. One of the most vital and proactive role of clinical pharmacist is the application of pharmacovigilance principles in day-to-day clinical practice. The establishment of pharmacovigilance surveillance schemes on use of pharmaceuticals is mandated by the substantial escalated diverse new drug-entities, presence of multiple co-morbidities and inappropriate and problematic poly-pharmacy.

Despite the availability of adverse drug reactions (ADR's) reporting systems, patient safety net and many data-bases; pharmacovigilance needs to be incorporated as part of this electronic data-base (not isolated) such as electronic prescribing, physician-decision matrix (order-entry) and patient profile systems. The advantage of such a trend is not just an added icon or drop-menu, it is a complete surveillance system that encompasses the essence, value and supportive nature of pharmacovigilance data blended with patient clinical health outcomes.

To keep the pace with the pharmaceuticals companies race, towards more new molecules with more benefit to patients' health; pharmacovigilance experts need to work hard to enforce the implementation of guidelines, policies and concepts of pharmacovigilance in more clinically-oriented pattern i.e. within the patient's health information system (clinical profile).

The colleges of pharmacy need to incorporate pharmacovigilance courses in their curriculum as the modest subjects of ADR's and drug safety. Research of pharmacovigilance nature will strengthen the clinical pharmacist's role in more clinically valued input.

The healthcare organizations and pharmacy societies worldwide have an important role to play in this regard. We urge for more collaboration between concerned parties to strengthen the clinical application of pharmacovigilance and to market this fundamental process in drug development, post-marketing and patient health outcome. 